\title{
Evaluation of antioxidant efficacy of Purslane extract in Patients with Recurrent Aphthous Stomatitis: a randomized, placebo-controlled, triple-blinded, clinical trial
}

\author{
Mahsa Mohammadzadeh ${ }^{1}$, Nima Rezaei ${ }^{2}$, Elnaz Kamran', Faraneh Abdolhoseinpour ${ }^{3}$, Mohsen Mohammadzadeh ${ }^{4}$ \\ Isaac Firooze Moqadam ${ }^{5}$,Shamsolmoulouk Najafi ${ }^{6,7}$, Mohsen Hoseini Ahmadabadi ${ }^{8}$ \\ ${ }^{1}$ Postgraduate student of orthodontics, Dental Branch, Islamic Azad University, Tehran, Iran \\ ${ }^{2}$ Research Center for Immunodeficiencies, Children's Medical Center, Tehran University of Medical Sciences, Tehran, Iran \\ ${ }^{3}$ Postgraduate student of pediatric dentistry, Dental Branch, Islamic Azad University, Tehran, Iran \\ ${ }^{4}$ D.D.S, MSc,Ophtalmologist, North-Khorasan university of medical sciences,Bojnourd,Iran.
}

${ }^{5}$ D.D.S, MSc, Periodontist, Private practice.

${ }^{6}$ Dental Research Center, School of Dentistry, Tehran University of Medical Sciences, Tehran,Iran. ${ }^{7}$ Department of Oral Medicine, School of Dentistry, Tehran University of Medical Sciences, Tehran, Iran.

${ }^{8}$ Chimist,pharmacy tehran university of medical sciences, Tehran,Iran.

Received for publication: April 7, 2017 Accepted: May 27, 2017

Corresponding author: Shamsolmolouk Najafi, DDS, MS Address: Department of Oral Medicine, School of Dentistry, Tehran University of Medical Sciences.

E-mail: najafi_drsh@yahoo.com Running Title: Purslane efficacy in treatment of Aphthous Stomatitis
Abstract

Background: This herbal medicine is considered a rich source of antioxidants with anti-inflammatory effects. The purpose of this study was to evaluate the effectiveness of purslane in treatment of recurrent aphthous stomatitis (RAS) and also it $\llbracket s$ effect on antioxidant level. Materials and methods: 50 patients were selected for this randomized triple-blind placebo-controlled trial. All subjects were randomly divided in to two groups, one group received purslane $(n=25)$ and another group, placebo ( $n=25)$ for 3 month. Superoxide dismutase (SOD), glutathione peroxidase (GSHPx) and total antioxidant status (TAS) was measured in plasma at baseline and after 3 month of treatment. Also pain intensity based on the visual analogue scale (VAS), the mean interval between lesion, number of lesions and the mean duration of complete healing at baseline and in month 1,2 and 3 were recorded. Statistical analysis was performed by using Mann-Whitney and T-test. Results: A significant decrease in pain intensity in VAS scores was seen after treatment in intervention group $(p<0.001)$. The mean duration of complete healing showed significant differences $(P<0.001)$ between the two groups. The mean interval between lesions also showed significant differences $(P<0.001)$ among the intervention group (33.12 days) compared with the placebo group (17.88 days). No significant differences were found regarding the number of lesions, level of erythrocyte GSHPx, TAS and SOD. No serious side-effects occurred in either of groups. Conclusions: According to this study, purslane is clinically effective in treatment of RAS (number of lesions, pain intensity and duration of healing) although it is unable to change the level of antioxidants.

Keywords: Recurrent aphthous stomatitis, Antioxidants, Purslane, Treatment

\section{Introduction}

One of the most common oral inflammatory ulcerative diseases, involving more than $20 \%$ of population worldwide, is recurrent aphthous stomatitis (RAS) ${ }^{1}$. The disease is 
characterized by painful, round or ovoid ulcers with circumscribed margins, white-gray pseudomembrane and erythematous haloes in non-keratinized mucosa specially the lips, the wall of the cheeks, soft palate and ventral surface of the tongue and floor of the mouth ${ }^{2}$. Three clinical forms of the disease has been recognized: minor (MiRAS), major (MaRAS) and herpetic form ulcers. MiRAS accounts for $80 \%$ of RAS patients ${ }^{3}$.

The etiology of RAS is unknown, but several immunologic, hematologic, allergic and psychologic disorders have been proposed as causative agents. Such malnutritions as iron, folic acid and B12 vitamin deficiencies, local trauma, emotional stress, hormonal changes, and infectious agents have also been suggested as etiologic factors ${ }^{2,4}$. Recently it has been proposed that free radicals may lead to RAS through oxidative stress pathway ${ }^{5}$. Decreasing antioxidant defenses in the body or increasing free radicals level, known as oxidative stress, has several destructive effects and often leads to tissue breakdown, which is proposed to play a significant role in development and progression of RAS 5 .

Purslane or Portulaca oleracea is from the Portulacaceae family, which possesses antioxidant and anti-inflammatory properties $^{6}$, and it has been clinically effective in the treatment of oral mucosal disorders such as oral lichen planus ${ }^{7}$. The aims of this study were to evaluate the efficacy of purslane in the treatment of RAS, and its antioxidant efficacy in the serum of patients with RAS.

\section{Materials and methods}

\section{Subjects and study design}

The present study was a randomized, triple blinded, placebocontrolled clinical trial. Fifty patients with MiRAS comprised of 22 male and 28 female (range from 19 to 55 years) from the Department of Oral Medicine, School of Dentistry, Tehran University of Medical Sciences were enrolled in this study based on the inclusion and exclusion criteria (Table 1).

Table 1 - Selection Criteria

Inclusion criteria
1. History of presenting at least three recurrences per year
2. Clinically being in active phase
3. Educated enough to understand the method and sign the informed consent
form (older than 18)
Exclusion criteria
1. History of any local or systemic non-aphthous diseases such as diabetes, hepa-
titis, HIV, blood pressure, cardiovascular or neurological or respiratory disorders
2. Treatment with immunosuppressive drugs 1 month prior to the study
3. Treatment with iron or vitamins 3 months prior to the study
4. Patients with Behcet disease and any other active inflammatory bullous diseases

All the patients were randomly divided into two groups (balance block randomized): 25 patients in intervention group and 25 patients in placebo group. Gender, age and medical history of patients were recorded. Research Ethics Committee of Tehran University of Medical Sciences approved the study. Written informed consent was obtained from all subjects.

\section{Plant collection and extraction}

We collected plants from the southern part of Tehran, capital city of Iran. It was authenticated in faculty of pharmacy, Tehran University of Medical Sciences. Aerial parts of the plant and some of the seeds were stabilized in boiling alcohol and were extracted by continuous extractor in environmental temperature with $96^{\circ} \mathrm{C}$ ethanol. This method effectively prevents any enzymatic destruction. The resulting extract was filtered by filter and centrifuge. Then a vaccume-distiller was used to produce a very viscose residue.

A dosage form was prepared and the extraction was granulated with an appropriate amount of lactose. The granules were filled in hard capsules, each containing $235 \mathrm{mg}$ extract, based on previous studies ${ }^{7}$. The capsules had the same color, with the same shape and size and were coded.

\section{Intervention}

Patients in intervention group received the capsules containing purslane extract, and patients in control group received the same capsules in shape, size and color but containing placebo. The subjects were told to take 2 capsules each day (in morning and at night) for 3 months. The investigator and subjects and analyzer were blind to the code record.

\section{Outcome measures}

The number of lesions, the mean interval between lesions (in days), and the mean duration of complete healing (in days), at baseline and in month 1,2 and 3 were measured. Moreover, the following outcome measures were recorded:

Pain Severity: To evaluate the severity of pain, a visual analogue scale (VAS) ranged from 0 , showing no pain; to 10 , showing extreme pain ${ }^{8}$ was used at baseline and in month1, 2 and 3.

Antioxidant Status: In brief, blood samples were collected from subjects based on venous puncture method at baseline and in month 1, 2 and 3 in order to detect the level of total antioxidant status (TAS) and suoer oxide dismutase (SOD) and glutathione peroxidase (GSHPx). The blood was centrifuged at $3000 \mathrm{rpm}$ for 5 minutes at . RANSEL kit was used to measure the activity rate of SOD. The activity rate of SOD was measured based on inhibition of the reaction between superoxide radical and lodophenyl nitrophenol phenyl tetrazoliu-chloride (INT) by SOD. One unit of enzyme was defined as the amount of enzyme that inhibits $50 \%$ of INT reduction rate. RANSEL kit was used to measure the activities of GSHPx, based on reduction in absorption degree at $340 \mathrm{~nm}$ (after the oxidation of NADPH). The measurement of TAS was based on spectrophotometry.

\section{Statistical analysis}

T-test and Mann-whitney test served for comparisons of pain severity, the mean interval between lesion occurrence, and serum level of antioxidant between the two groups. Statistical significance was set at 0.05 .

\section{Results}

A significant difference existed in pain severity between the two groups after 3 months, with more pain relief occurring in 
intervention group $(\mathrm{p}<0.001)$ (Table 2).

Table 2 - Comparison of pain relief between the two groups

\begin{tabular}{lll}
\hline Pain relief & Placebo (\%) & Intervention (\%) \\
\hline 1 ( 1 degree worsening) & 4 & 0 \\
0 ( no change) & 48 & 4 \\
-4 ( 4 degrees improvement) & 28 & 40 \\
-3 ( 3 degrees improvement) & 8 & 20 \\
-2 ( 2 degrees improvement) & 8 & 20 \\
-1 ( 1 degree improvement) & 4 & 16 \\
\hline
\end{tabular}

Patients in the intervention group experienced significantly longer interval between lesion recurrence compared to the patients in control group at 3 months follow up (33.12 vs. 17.88 days respectively, $p<0.001$ ) (Table 3 ). At the same follow up, the decrease in healing duration was also significantly more among patients in intervention group compared to that in patients in control group $(6.56 \pm 4.50$ vs. $1.52 \pm 4.07$ day respectively, $\mathrm{p}<0.001$ ) (Table 3).

The differences in number of lesions (Table 3), TAS levels, GSHPx levels, and SOD levels (Table 4) between the two groups at 3 months follow up remained insignificant ( $p>0.05)$.

Table 3 - Comparison of number of lesions, healing duration and interval between lesions recurrences

\begin{tabular}{llll}
\hline & Intervention & Placebo & $\mathrm{P}$ value \\
\hline $\begin{array}{l}\text { Healing } \\
\text { duration }\end{array}$ & $6.56 \pm 4.50$ & $1.52 \pm 4.07$ & $\mathrm{P}<0.001^{*}$ \\
$\begin{array}{l}\text { Interval } \\
\text { between }\end{array}$ & 33.12 & 17.88 & $\mathrm{P}<0.001^{*}$ \\
lesions & & & \\
$\begin{array}{l}\text { Number of } \\
\text { lesions }\end{array}$ & $1.28 \pm 1.07$ & $0.8 \pm 1.65$ & $\mathrm{P}=0.23$ \\
\hline
\end{tabular}

Table 4 - Comparison of mean and standard deviation of TAS, SOD and GSHPx between the two groups

\begin{tabular}{llll} 
& $\begin{array}{l}\text { Case } \\
\mathrm{N}=25\end{array}$ & $\begin{array}{l}\text { Control } \\
\mathrm{N}=25\end{array}$ & P value \\
\hline TAS & $0.01 \pm 0.23$ & $0.04 \pm 0.2$ & 0.62 \\
GSHPx & $18.87 \pm 29.78$ & $19.20 \pm 53.31$ & 0.98 \\
SOD & $14.6 \pm 48.91$ & $42.44 \pm 70.53$ & 0.11 \\
\hline
\end{tabular}

\section{Discussion}

Studies have shown that RAS prevalence is higher among women than men ${ }^{9}$ especially between the ages of 20 to 30 years ${ }^{4}$. In our study the average age of participants was 35 years, and the majority of them were women ( 28 women vs. 22 men).

Although Azizi et al. ${ }^{10}$ and Gudaz et al. ${ }^{11}$ found no significant differences in antioxidant levels between RAS patients and control group ${ }^{10,11}$, disorders in antioxidant system in patients with RAS has been reported by several studies ${ }^{1,12-14}$.

To the best of our knowledge, the present study is the first triple-blind placebo-controlled clinical trial on efficacy of antioxidant-rich purslane and its anti-inflammatory effects ${ }^{15,16}$ in treatment of RAS. According to the results, no significant changes in the levels of SOD, GSHPx, and TAS occurred in the purslanetreated patients. However, our study demonstrated a beneficial effect of purslane in healing or controlling symptoms of RAS. This effect might be related to other components of this plant, such as: phosphate, zinc, iron, etc. Efficacy of various drugs in treatment of RAS such as rebamipide, colchicine and petonxyfilline has been studied previously ${ }^{9,17}$. None of them, however, has been as effective as purslane. However, many adverse side effects were reported for colchicine and talidomide ${ }^{17-19}$, none of which occurred in purslane-treated patients up to 3 months of treatment.

In the present study we demonstrated that an oral tablet with purslane can reduce pain severity, healing duration, and can increase the interval between lesions recurrences with no adverse side effects at 3 months follow up. Further studies on larger groups of patients with longer follow-up might be needed to confirm these results.

\section{Aknowledgement}

The authors are very thankful to all patients and their families for their kind collaboration in this study. This research has been supported by Tehran University of Medical Sciences, faculty of Dentistry.

\section{Conflict Of Interest}

The authors declare that they have no conflict of interests.

\section{References}

1. Momen-Beitollahi J, Mansourian A, Momen-Heravi F, Amanlou M, Obradov S, Sahebjamee M. Assessment of salivary and serum antioxidant status in patients with recurrent aphthous stomatitis. Med Oral patol Oral cir bucal. $2010 \mathrm{Jul}$ 1;15(4):e557-61.

2. Koybasi S, Parlak AH, Serin E, Yilmaz F, Serin D. Recurrent aphthous stomatitis: investigation of possible etiologic factors. Am J Otolaryngol. 2006 Jul-Aug;27(4):229-32.

3. Altenburg A, Zouboulis CC. Current concepts in the treatment of recurrent aphthous stomatitis. Skin therapy lett. 2008 Sep;13(7):1-4.

4. Jurge S, Kuffer R, Scully C, Porter SR. Number VI Recurrent aphthous stomatitis. Oral Dis. 2006 Jan 1;12(1):1-21.

5. Gurel A, Altinyazar HC, Unalacak M, Armutcu F, Koca R. Purine catabolic enzymes and nitric oxide in patients with recurrent aphthous ulceration. Oral Dis. 2007 Nov 1;13(6):570-4.

6. Movahedian A, Ghannadi A, Vashirnia M. Hypocholesterolemic effects of purslane extract on serum lipids in rabbits fed with high cholesterol levels. Int J Pharmacol. 2007;3(3):285-9.

7. Agha Hosseini F, Borhan Mojabi K, Monsef Esfahani HR, Mirzaii Dizgah I, Etemad Moghadam S, Karagah A. Efficacy of purslane in the treatment of oral lichen planus. Phytotherapy research. 2010 Feb 1;24(2):240-4.

8. Scott J, Huskisson EC. Graphic representation of pain. Pain. 1976 Jun 1;2(2):175-84.

9. Mimura MA, Hirota SK, Sugaya NN, Sanches Jr JA, Migliari DA. Systemic treatment in severe cases of recurrent aphthous stomatitis: an open trial. Clinics. 2009 Mar;64(3):193-8. 
10. Azizi A, Shah Siah S, Madhani A. Comparison of amount of salivary total antioxidant in patients with recurrent aphtous stomatitis. J Dent Med. 2012 Apr 15;25(1):14-8.

11. Gunduz K, Ozturk G, Sozmen EY. Erythrocyte superoxide dismutase, catalase activities and plasma nitrite and nitrate levels in patients with Behcet disease and recurrent aphthous stomatitis. Clinical and experimental dermatology. 2004 Mar 1;29(2):176-9.

12. Saral $Y$, Coskun BK, Ozturk $P$, Karatas F, Ayar A. Assessment of salivary and serum antioxidant vitamins and lipid peroxidation in patients with recurrent aphthous ulceration. The Tohoku journal of experimental medicine. 2005;206(4):305-12.

13. Karincaoglu Y, Batcioglu K, Erdem T, Esrefoglu M, Genc M. The levels of plasma and salivary antioxidants in the patient with recurrent aphthous stomatitis. J Oral Pathol Med. 2005 Jan 1;34(1):7-12.

14. Arikan S, Durusoy C, Akalin N, Haberal A, Seckin D. Oxidant/antioxidant status in recurrent aphthous stomatitis. Oral Dis. 2009 Oct
$1 ; 15(7): 512-5$.

15. Ezekwe MO, Omara-Alwala TR, Membrahtu T. Nutritive characterization of purslane accessions as influenced by planting date. Plant Foods Hum Nutr. 1999;54(3):183-91.

16. Simopoulos AP, Norman HA, Gillaspy JE, Duke JA. Common purslane: a source of omega-3 fatty acids and antioxidants. J Am Coll Nutr. 1992 Aug;11(4):374-82.

17. Liu C, Zhou Z, Liu G, Wang Q, Chen J, Wang L, et al. Efficacy and safety of dexamethasone ointment on recurrent aphthous ulceration. Am J Med. 2012 Mar 31;125(3):292-301.

18. Stephen J. Challacombe, Surab Alsahaf, Anwar Tappuni. Recurrent Aphthous Stomatitis: Towards Evidence-Based Treatment?. Curr Oral Health Rep. 2015. 2: 158.

19. Leung YY, Hui LL, Kraus VB. Colchicine-Update on mechanisms of action and therapeutic uses. InSeminars in arthritis and rheumatism 2015 Dec 31: 45(3): 341-350. 